\title{
IMPACTO DE DIFERENTES CICLOS DE EMISSÕES EM UM VEÍCULO FLEX-FUEL
}

\author{
Eduardo Costa Quadros \\ Continental Indústria Automotiva LTDA \\ Email: eduardo.quadros@ continental-corporation.com
}

\section{RESUMO}

Com a crescente preocupação com o meio ambiente e o constante avanço das legislações de emissões mundiais, a introdução de novos ciclos de emissões e procedimentos para testes é uma tendência. A legislação brasileira segue o padrão norte americano e usa o FTP75 para homologação de seus veículos. Porém, cada vez mais, a representatividade dos ciclos de emissões é questionada. Este estudo tem como objetivo testar o mesmo veículo em diferentes ciclos de emissões (FTP75, NEDC e WLTC) e analisar as diferenças de emissões de gases de escape e de um veículo flex-fuel, que atende ao PROCONVE L6. Ao fim do estudo, indica-se se a legislação brasileira, com o uso do FTP75, está conseguindo seguir os níveis de emissões internacionais, ou se a mesma está ficando defasada em relação aos padrões internacionais.

\section{INTRODUÇÃO}

Para a realização deste estudo foram feitos testes em diferentes ciclos de emissões com um veículo em produção que atende as normas vigentes no país. O objetivo é verificar diferenças entre ciclos e influências de combustíveis utilizados para homologação no Brasil: A22H0 (E22) e EHR (E100).

Os três ciclos escolhidos foram o Federal Test Procedure (FTP75), o New European Driving Cycle (NEDC) e o Worldwide harmonized Light vehicles Test Cycle (WLTC). Para cada ciclo foram feitos três ensaios de emissões completos. 


\section{CARACTERÍSTICAS DO VEÍCULO}

Para os testes, foi utilizado um veículo em produção no país. O mesmo atende os limites do PROCONVE L6, e possui um motor flex-fuel 1.61 de quatro cilindros com transmissão automática. Os testes foram realizados com motor e catalisador com cerca de $10.000 \mathrm{~km}$.

\section{CARACTERÍSTICAS DOS CICLOS}

Os três ciclos abordados neste estudo são: o FTP75 (Federal Test Procedure), o NEDC (New European Driving Cycle) e o WLTC classe 3 (Worldwide harmonized Light vehicles Test Cycle). O FTP75 é o ciclo norte-americano utilizado atualmente para homologação de veículos no Brasil. O NEDC, ciclo europeu, é referência não só na Europa, mas também em algumas outras regiões, como China e América Latina. Há algum tempo, o NEDC é questionado pelo seu perfil de velocidades, que é em sua maioria constante, e não se aproxima de um uso real do veículo. Para cobrir essa deficiência do NEDC, foi criado o WLTC, que agora também é necessário para homologações de veículos na Europa. As principais características dos três ciclos estão descritas na Tabela 1.

Tabela 1: Principais características do FTP75, NEDC e WLTC [1],[2].

\begin{tabular}{|l|c|c|c|}
\cline { 2 - 4 } \multicolumn{1}{c|}{} & FTP75 & NEDC & WLTC \\
\hline Duração (s) & 1874 & 1180 & 1800 \\
\hline Distância $(\mathrm{km})$ & 17.77 & 11.03 & 23.27 \\
\hline Velocidade Média (km/h) & 34.1 & 33.6 & 46.5 \\
\hline Velocidade Máxima (km/h) & 91.2 & 120 & 131.3 \\
\hline Aceleração Positiva Média (m/s $)$ & 0.51 & 0.59 & 0.41 \\
\hline Aceleração Positiva Máxima (m/s $)$ & 1.48 & 1.04 & 1.67 \\
\hline Tempo parado (s) e (\%) & $357(19.1 \%)$ & $280(23.7 \%)$ & $226(12.6 \%)$ \\
\hline Tempo em velocidade constante (s) e (\%) & $123(6.6 \%)$ & $475(40.3 \%)$ & $66(3.7 \%)$ \\
\hline Tempo em aceleração (s) e (\%) & $739(39.4 \%)$ & $247(20.9 \%)$ & $789(43.8 \%)$ \\
\hline
\end{tabular}

O WLTC destaca-se por ser o ciclo com maior distância, maior velocidade média, maior velocidade máxima, maior aceleração positiva máxima, menor tempo parado, menor tempo em velocidade constante e maior tempo em aceleração. Estas características fazem deste ciclo, o mais severo e possivelmente o mais realista entre os três. 
Os limites de emissões para cada um dos ciclos estão representados em g/km na Tabela 2. Seguindo os padrões do PL6 para o FTP75, e EURO 6 para o NEDC e WLTC:

Tabela 2: Limites de emissões PL6 e EURO6 [3].

\begin{tabular}{|l|c|c|c|}
\cline { 2 - 4 } \multicolumn{1}{c|}{} & FTP75 & NEDC & WLTC \\
\hline THC $(\mathrm{g} / \mathrm{km})$ & 0.3 & 0.1 & 0.1 \\
\hline NMHC $(\mathrm{g} / \mathrm{km})$ & 0.05 & 0.068 & 0.068 \\
\hline CO $(\mathrm{g} / \mathrm{km})$ & 1.3 & 1.0 & 1.0 \\
\hline NOx $(\mathrm{g} / \mathrm{km})$ & 0.08 & 0.06 & 0.06 \\
\hline CHO $(\mathrm{g} / \mathrm{km})$ & 0.02 & - & - \\
\hline
\end{tabular}

Além dos dados acima, é importante citar também fatores relevantes para a fase fria de cada um dos ciclos. Como mostra a Tabela 3, a fase fria (primeira fase) do FTP75 tem peso 0.43 no resultado final. No NEDC e WLTC não há fator de ponderação entre as fases, ou seja, o resultado em $\mathrm{g} / \mathrm{km}$ final será a soma das emissões em massa de todas as fases dividida pela soma da distância de todas as fases. Outro fator importante relacionado à fase fria é o tempo em marcha lenta antes da primeira aceleração. No NEDC e WLTC este tempo é de 11 segundos. Já no FTP75 ele sobe para 20 segundos. Este tempo a mais é importante, pois é utilizado para aquecer o catalisador antes da primeira aceleração.

Tabela 3: Dados importantes que influenciam a fase fria dos ciclos

\begin{tabular}{|l|l|l|l|}
\cline { 2 - 4 } \multicolumn{1}{c|}{} & \multicolumn{1}{c|}{ FTP75 } & \multicolumn{1}{c|}{ NEDC } & \multicolumn{1}{c|}{ WLTC } \\
\hline Tempo antes da aceleração & 20 segundos & 11 segundos & 11 segundos \\
\hline Peso (fator de ponderação) & 0.43 & 1 & 1 \\
\hline
\end{tabular}

Para o FTP75 foram utilizados dados de inércia com adição de $10 \%$, devido à norma brasileira. Para os ciclos NEDC e WLTC, foram usados os dados originais do veículo.

Na Figura 1: Pontos de operação dos três ciclos: Rotação em RPM no eixo x, e massa de ar por tempo (mg/str) no eixo yFigura 1, estão representados os pontos de operação dos três ciclos. Observase que o NEDC é o ciclo com menor número de pontos em altas cargas, e o WLTC é o ciclo com maior número de pontos em altas cargas. Além disso, o WLTC é também o ciclo que atinge a maior rotação máxima do motor, pouco acima dos $4000 \mathrm{rpm}$. 


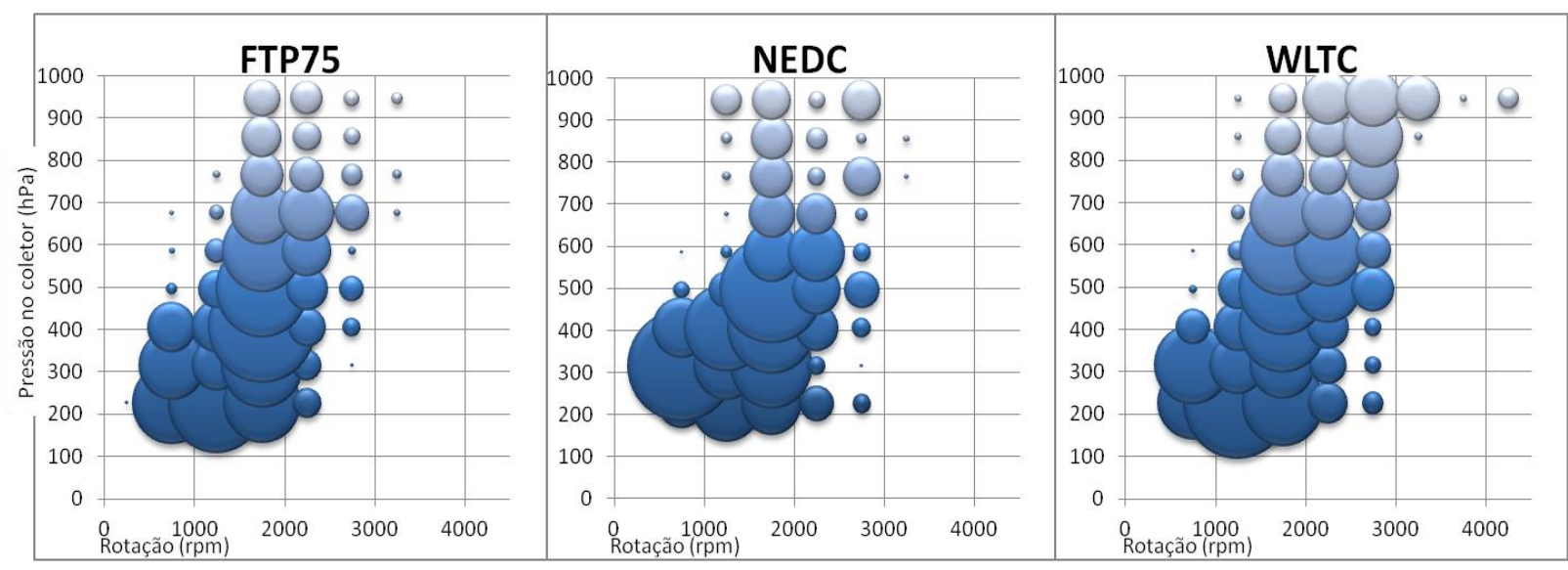

Figura 1: Pontos de operação dos três ciclos: Rotação em RPM no eixo x, e massa de ar por tempo (mg/str) no eixo y

\section{RESULTADOS}

\subsection{RESULTADOS COM E22}

As médias dos resultados ponderados dos três FTP75 com E22 encontram-se na Tabela 4. Os valores representados abaixo são resultados das médias entre os três testes realizados em cada ciclo. Os resultados estão representados em $\mathrm{g} / \mathrm{km}$ e em função dos limites de cada um dos ciclos.

Tabela 4: Resultados ponderados dos testes com E22 em g/km e em função dos limites de cada uma das legislações

\begin{tabular}{|c|c|c|c|c|c|c|}
\hline & \multicolumn{2}{|c|}{ FTP75 } & \multicolumn{2}{|c|}{ NEDC } & \multicolumn{2}{|c|}{ WLTC } \\
\hline & $\begin{array}{l}\text { Total } \\
(\mathrm{g} / \mathrm{km})\end{array}$ & $\begin{array}{c}\text { Limite } \\
(\%)\end{array}$ & $\begin{array}{l}\text { Total } \\
(\mathrm{g} / \mathrm{km})\end{array}$ & $\begin{array}{c}\text { Limite } \\
(\%)\end{array}$ & $\begin{array}{l}\text { Total } \\
(\mathrm{g} / \mathrm{km})\end{array}$ & $\begin{array}{c}\text { Limite } \\
(\%)\end{array}$ \\
\hline THC & 0.032 & $10.7 \%$ & 0.072 & $72.0 \%$ & 0.044 & $43.7 \%$ \\
\hline NMHC & 0.028 & $56.0 \%$ & 0.067 & $98.5 \%$ & 0.040 & $58.3 \%$ \\
\hline $\mathrm{CO}$ & 0.183 & $14.1 \%$ & 0.383 & $38.3 \%$ & 0.316 & $31.6 \%$ \\
\hline NOx & 0.012 & $15.4 \%$ & 0.017 & $27.8 \%$ & 0.020 & $33.3 \%$ \\
\hline $\mathrm{CO} 2$ & \multicolumn{2}{|c|}{172.141} & \multicolumn{2}{|c|}{169.385} & \multicolumn{2}{|c|}{156.031} \\
\hline $\begin{array}{l}\text { Consumo } \\
(\mathrm{km} / \mathrm{l})\end{array}$ & \multicolumn{2}{|c|}{12.60} & \multicolumn{2}{|c|}{12.77} & \multicolumn{2}{|c|}{13.91} \\
\hline
\end{tabular}


Estes mesmo valores são representados graficamente na Figura 2. Em todos os ciclos, o gás mais crítico com o NMHC. No FTP75 e no WLTC, os valores atingidos estão bem abaixo dos limites (56.0 e 58.3\% respectivamente). Entretanto, no NEDC o valor de NMHC foi de 67 $\mathrm{mg} / \mathrm{km}$, ficando muito próximo do limite de $68 \mathrm{mg} / \mathrm{km}$.

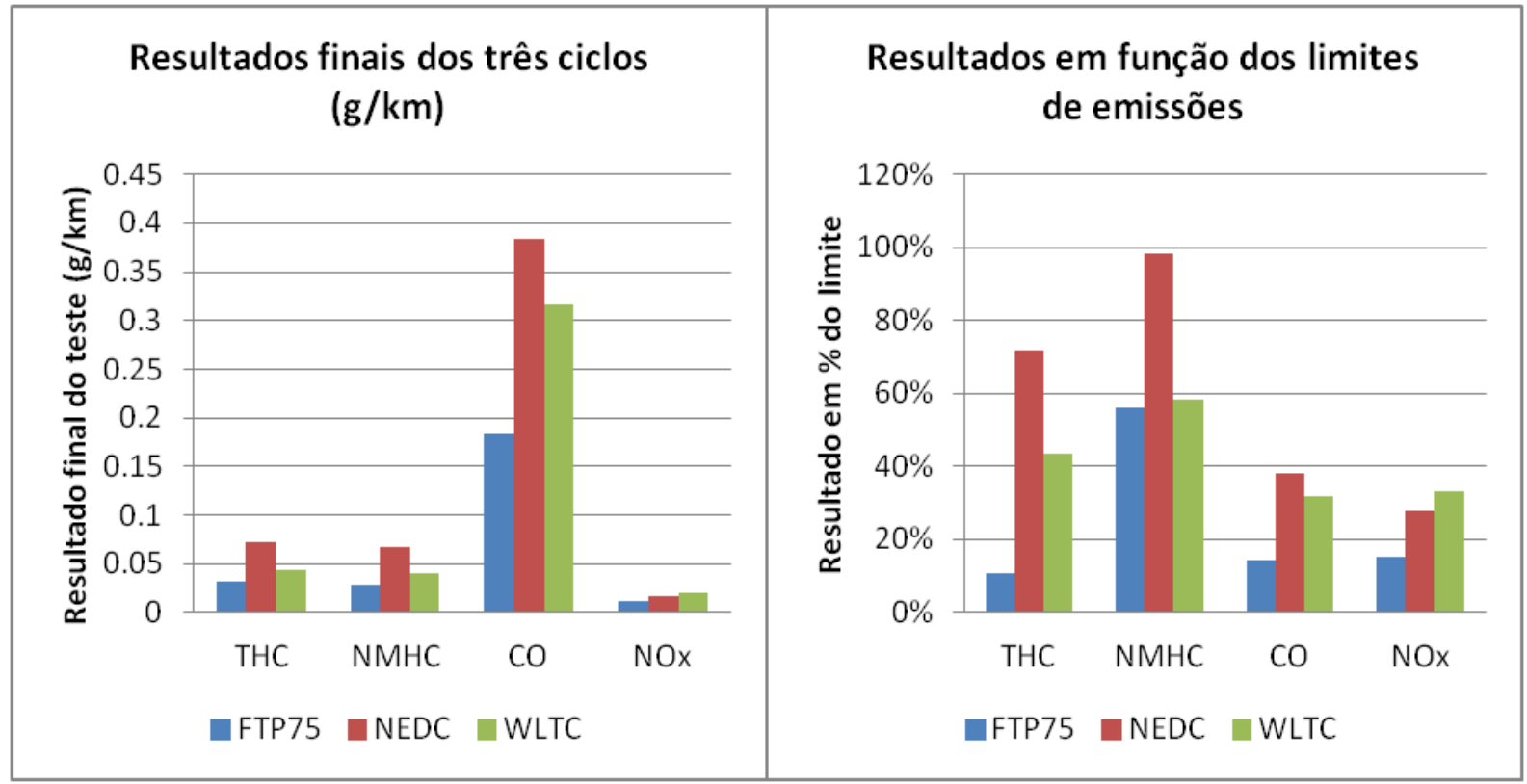

Figura 2: Representação gráfica dos resultados com E22

Além disso, os valores emitidos de CO e NOx elevaram-se no NEDC e WLTC em relação ao FTP75. Apesar da ligeira elevação, os valores ainda encontram-se consideravelmente abaixo dos limites para estes gases em todos os ciclos.

Apesar da aparente piora dos resultados em relação ao FTP75, grande parte dessas diferenças se deve aos diferentes fatores de ponderação entre os ciclos. Como destacado na Tabela 3, a fase fria do FTP75 (fase 1) representa uma parcela reduzida do resultado final em relação ao NEDC e WLTC. Excluindo este peso diferente entre fases, os resultados do FTP75 pioram significantemente. Estas diferenças estão representadas na Figura 3. A diferença mais significativa em $\mathrm{g} / \mathrm{km}$ é do $\mathrm{CO}$, mas mesmo assim, esse gás ainda fica um tanto quanto abaixo dos limites legais. O NMHC também aumenta bastante, e passa de $56 \%$ para $83.4 \%$ do limite legal. 


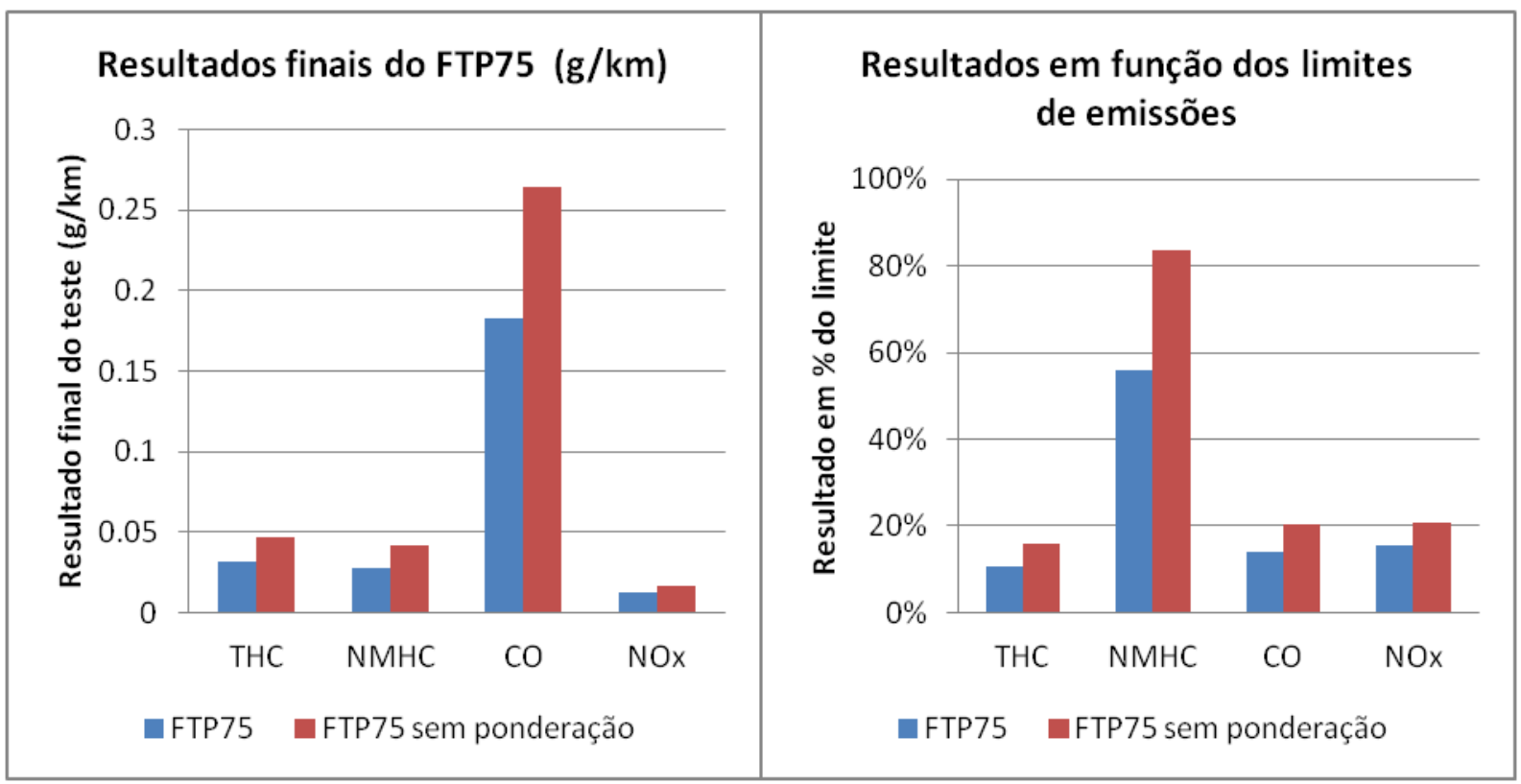

Figura 3: Comparação de resultados do FTP75 com e sem fatores de ponderação por fase

A Figura 4 mostra os sinais dos analisadores de THC durante os três ciclos. Nela, percebe-se que a maior parte de THC é gerada na primeira fase de cada ciclo, que inclui partida a frio e as primeiras acelerações.

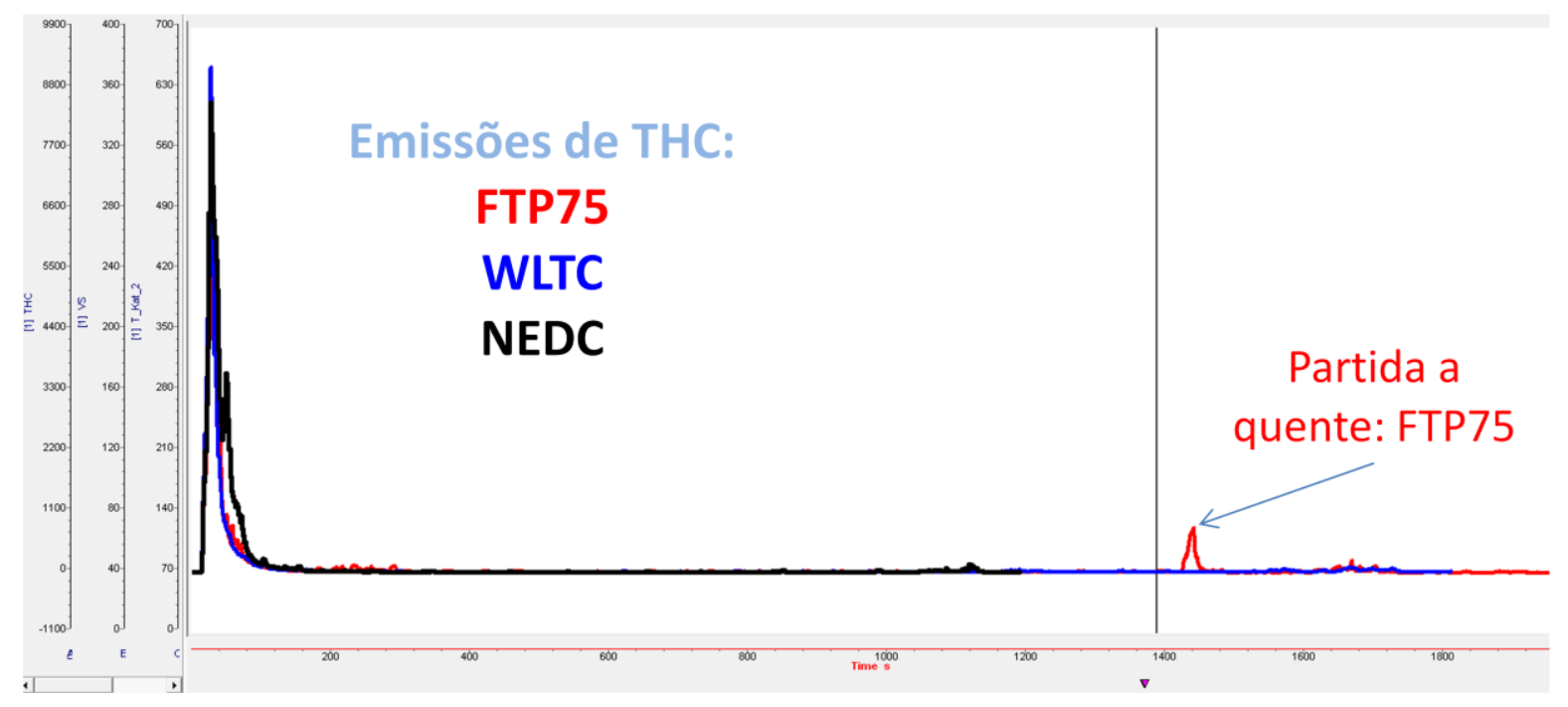

Figura 4: Comparação de modal entre os três ciclos

Após aproximadamente 200 segundos de teste, praticamente não há mais emissões de HC. Na Figura 5, o detalhe do início do teste evidencia este fato. Nota-se também que a concentração de THC no NEDC demora mais tempo para diminuir, pois devido às condições do ciclo, o catalisador demora mais tempo para ser aquecido. 


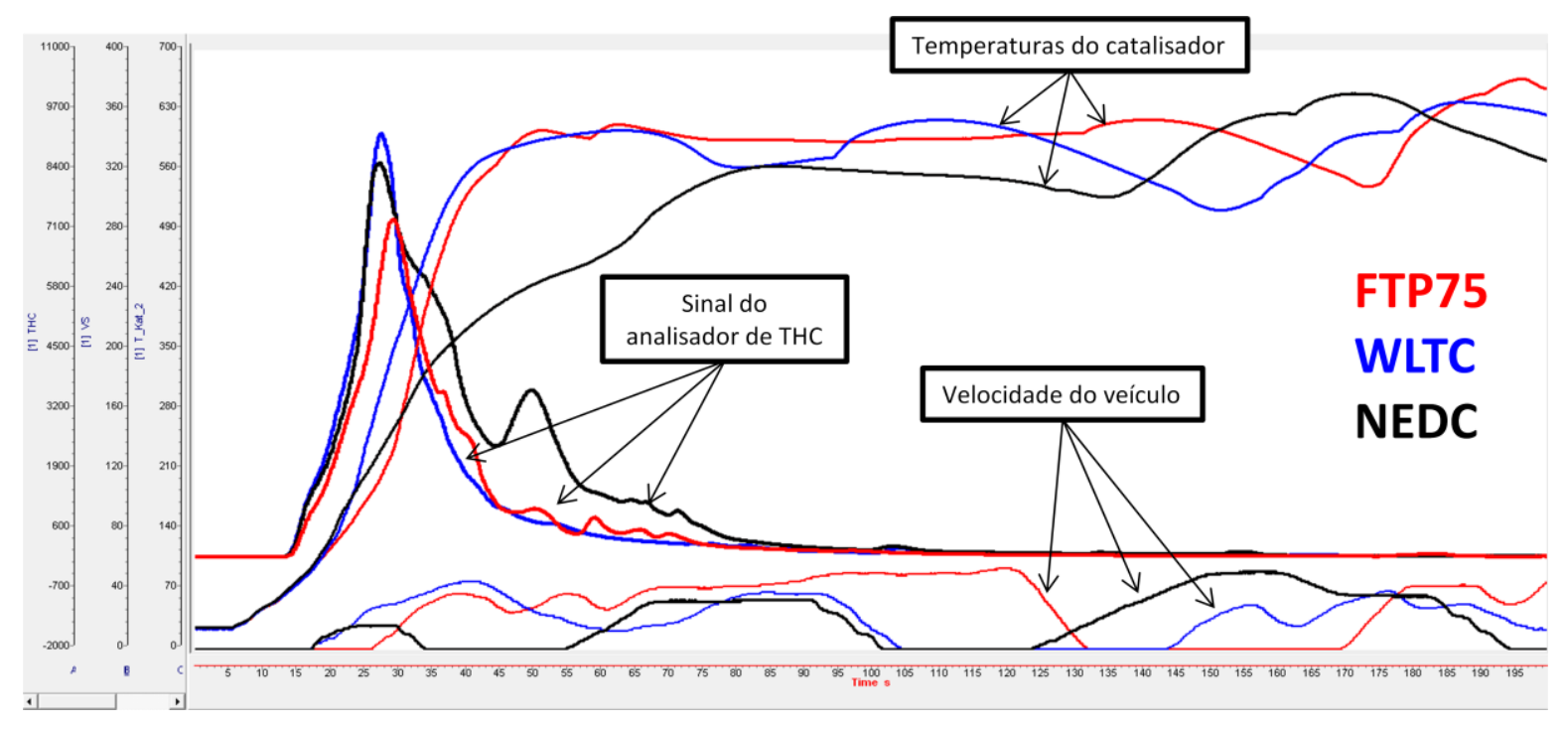

Figura 5: Comparação do modal entre os três ciclos nos primeiros 200 segundos de teste

Dado que a maior parte dos gases poluentes é emitida nos primeiros segundos dos testes, faz sentido analisar as emissões totais em massa, e não em gramas por quilômetro. Na Tabela 5 abaixo, são comparados às emissões totais em massa na fase fria de cada ciclo (Fase I) e na(s) fase(s) quente(s) (II no NEDC, II e III no FTP75 e II, III e IV no WLTC).

Tabela 5: Comparação de emissões em massa nas fases frias e quentes

\begin{tabular}{|l|c|c|c|c|c|c|}
\cline { 2 - 7 } \multicolumn{1}{c|}{} & \multicolumn{3}{c|}{ Fase Fria (I) } & \multicolumn{3}{c|}{ Fase Quente (II, III, IV) } \\
\cline { 2 - 7 } \multicolumn{1}{c|}{} & FTP75 & NEDC & WLTC & FTP75 & NEDC & WLTC \\
\hline Distância $(\mathrm{km})$ & 5.776 & 3.957 & 3.081 & 11.952 & 6.877 & 20.041 \\
\hline THC $(\mathrm{g})$ & 0.724 & 0.727 & 0.802 & 0.111 & 0.054 & 0.207 \\
\hline NMHC $(\mathrm{g})$ & 0.666 & 0.682 & 0.748 & 0.073 & 0.042 & 0.165 \\
\hline CO $(\mathrm{g})$ & 3.612 & 3.609 & 4.001 & 1.078 & 0.535 & 3.312 \\
\hline NOx $(\mathrm{g})$ & 0.220 & 0.127 & 0.367 & 0.074 & 0.052 & 0.089 \\
\hline
\end{tabular}

No comparativo nota-se que as emissões de HC, NMHC e CO nas fases frias são semelhantes entre os três ciclos. Isto evidencia que a estratégia de aquecimento de catalisador adotada funciona também em ciclos diversos, não apenas no FTP75. O WLTC têm valores ligeiramente maiores que o FTP75 e o NEDC. Isto acontece devido ao menor tempo antes da aceleração (em relação ao FTP75) e à maior velocidade máxima da primeira rampa no WLTC. 
O caso do NMHC, que foi o mais crítico, está ilustrado na Figura 6. Esta destaca a pequena diferença nas emissões na primeira fase entre cada um dos ciclos.

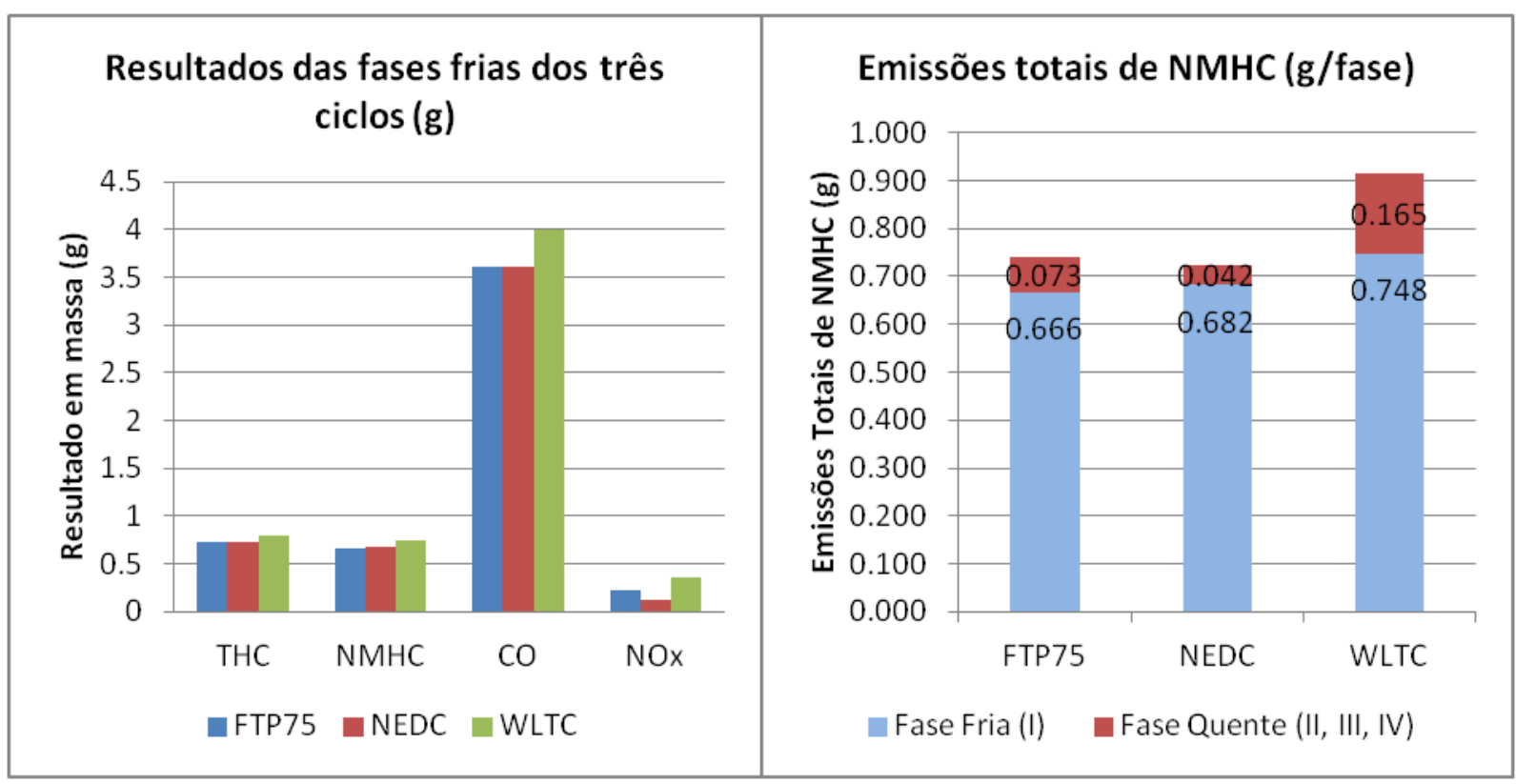

Figura 6: Resultados das fases frias em massa e comparação de emissões de NMHC nas fase frias e quentes.

Os resultados em massa são semelhantes, mas como os resultados finais dos testes são dados em $\mathrm{g} / \mathrm{km}$, a distância de cada ciclo e os fatores de ponderação por fase tem mais influência no resultado final do que a massa de poluentes emitida de fato durante os testes.

Nas fases quentes, o WLTC também foi o ciclo com maiores emissões. Os valores de HC e NMHC foram praticamente o dobro que os valores do FTP75 e as emissões de CO foram quase 3 vezes maiores. Na Tabela 6, estão descritas as proporções das primeiras fases de cada ciclo em relação ao ciclo inteiro. Nela, nota-se que o NMHC emitido durante a primeira fase do FTP75, NEDC e WLTC representa respectivamente 90.1\%, 94.2\% e 81.9\%.

Tabela 6: Proporção de massa emitida durantes fases frias

\begin{tabular}{|l|c|c|c|}
\cline { 2 - 4 } \multicolumn{1}{c|}{} & \multicolumn{3}{c|}{ Proporção da fase fria sobre os ciclos inteiros } \\
\cline { 2 - 4 } \multicolumn{1}{c|}{} & FTP75 & NEDC & WLTC \\
\hline Distância $(\mathrm{km})$ & $32.6 \%$ & $36.5 \%$ & $13.3 \%$ \\
\hline THC $(\mathrm{g})$ & $86.7 \%$ & $93.1 \%$ & $79.5 \%$ \\
\hline NMHC $(\mathrm{g})$ & $90.1 \%$ & $94.2 \%$ & $81.9 \%$ \\
\hline CO (g) & $77.0 \%$ & $87.1 \%$ & $54.7 \%$ \\
\hline NOx $(\mathrm{g})$ & $74.9 \%$ & $70.9 \%$ & $80.5 \%$ \\
\hline
\end{tabular}


Em todos os ciclos, a grande maioria das emissões acontece na fase fria do teste. Dentre os três ciclos, o WLTC é o ciclo que possui maiores emissões fora da fase fria. Entretanto, este é também o ciclo com maior quilometragem. Por causa das grandes diferenças de distância das fases quentes, e pelo fato de o catalisador já estar aquecido, portanto, trabalhando com alta eficiência, faz sentido comparar os valores em $\mathrm{g} / \mathrm{km}$ das fases quentes. Estes estão expressos na Tabela 7 .

Tabela 7: Resultados em massa e em $\mathrm{g} / \mathrm{km}$ das fases quentes

\begin{tabular}{|l|c|c|c|c|c|c|}
\cline { 2 - 7 } \multicolumn{1}{c|}{} & \multicolumn{3}{|c|}{ Fase Quente (II, II, IV) - (g) } & \multicolumn{2}{c|}{ Fase Quente (II, III, IV) - (g/km) } \\
\cline { 2 - 7 } \multicolumn{1}{c|}{} & FTP75 & NEDC & WLTC & FTP75 & NEDC & WLTC \\
\hline THC & 0.111 & 0.054 & 0.207 & 0.009 & 0.008 & 0.010 \\
\hline NMHC & 0.073 & 0.042 & 0.165 & 0.006 & 0.006 & 0.008 \\
\hline CO & 1.078 & 0.535 & 3.312 & 0.090 & 0.078 & 0.165 \\
\hline NOx & 0.074 & 0.052 & 0.089 & 0.006 & 0.008 & 0.004 \\
\hline
\end{tabular}

Os valores expressos em gramas por quilômetro da fase quente sinalizam leve tendência a maior geração de HC e NMHC no WLTC, e uma forte tendência à maior formação de CO em relação aos outros ciclos. A elevada emissão de CO acontece na quarta fase do WLTC, cuja velocidade máxima ultrapassa os $130 \mathrm{~km} / \mathrm{h}$. Este fato reforça a afirmação que o WLTC é o ciclo mais severo dentre os três.

\subsection{RESULTADOS COM E100}

As médias dos resultados ponderados dos três FTP75 com E100 encontram-se na

Tabela 8. Os valores representados abaixo são resultados das médias entre os três testes realizados em cada ciclo. Os resultados estão representados em $\mathrm{g} / \mathrm{km}$ e em função dos limites de cada um dos ciclos. Nos testes com E100, foram coletados também os aldeídos (CHO) e álcool não queimado. Para os ciclos NEDC e WLTC foi utilizada a metodologia de cálculo de NMHC com desconto de álcool não queimado (NMHC-ETOH) descrita na NBR 6601[4]. 
Tabela 8: Resultados em g/km e em parcela dos limites de emissões com E100

\begin{tabular}{|l|c|c|c|c|c|c|}
\cline { 2 - 7 } \multicolumn{1}{c|}{} & \multicolumn{2}{c|}{ FTP75 } & \multicolumn{2}{c|}{ NEDC } & \multicolumn{2}{c|}{ WLTC } \\
\cline { 2 - 7 } \multicolumn{1}{c|}{} & $\begin{array}{c}\text { Total } \\
(\mathrm{g} / \mathrm{km})\end{array}$ & $\begin{array}{c}\text { Limite } \\
(\%)\end{array}$ & $\begin{array}{c}\text { Total } \\
(\mathrm{g} / \mathrm{km})\end{array}$ & $\begin{array}{c}\text { Limite } \\
(\%)\end{array}$ & $\begin{array}{c}\text { Total } \\
(\mathrm{g} / \mathrm{km})\end{array}$ & $\begin{array}{c}\text { Limite } \\
(\%)\end{array}$ \\
\hline THC & 0.093 & $31.0 \%$ & 0.242 & $242.2 \%$ & 0.115 & $115.4 \%$ \\
\hline NMHC & 0.066 & $131.6 \%(1)$ & 0.187 & $275.7 \%$ & 0.085 & $125.7 \%$ \\
\hline NMHC-ETOH & 0.023 & $45.3 \%$ & 0.064 & $94.1 \%(2)$ & 0.032 & $46.6 \%$ \\
\hline NOx & 0.345 & $26.5 \%$ & 0.900 & $90.0 \%$ & 0.382 & $38.2 \%$ \\
\hline CHO & 0.010 & $13.0 \%$ & 0.017 & $27.5 \%$ & 0.015 & $25.0 \%$ \\
\hline CO2 (g/km) & 0.012 & $57.5 \%$ & 0.022 & $111.2 \%$ & 0.016 & $79.3 \%$ \\
\hline Consumo & 168.940 & \multicolumn{2}{c|}{152.309} & & 152.080 \\
$(\mathrm{~km} / \mathrm{l})$ & 8.46 & \multicolumn{2}{c|}{8.85} & & \\
\hline
\end{tabular}

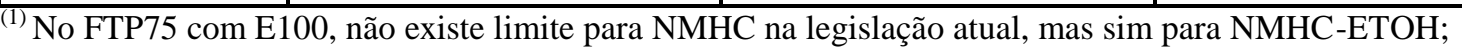

${ }^{(2)}$ No NEDC e WLTC não existem limites de NMHC-ETOH, nem de CHO. Para efeito de comparação do NMHC-ETOH e CHO, foram utilizados respectivamente o limite de NMHC do EURO 6 (0.068 g/km) e o limite de CHO do PL6 (0.020 g/km).

Segundo resultados dos testes, o veículo atende sem problemas à norma brasileira. $\mathrm{O}$ resultado do NMHC ficaria acima do limite do PL6 $(0.05 \mathrm{~g} / \mathrm{km})$, mas como é permitido descontar o valor de álcool não queimado, o resultado a ser comparado com o limite, é o do NMHC-ETOH.

No NEDC os resultados excederam os limites de THC e NMHC. Estes valores ficaram respectivamente $142 \%$ e $175 \%$ acima dos limites legais para estes gases. Aplicando o desconto de álcool não queimado, ainda seria possível respeitar o limite de NMHC, mas com uma margem muito pequena. O valor de $\mathrm{CHO}$ também ficou acima do limite fictício adotado para comparação. Além disso, o CO foi bem elevado, mas ainda ficou abaixo do limite com uma margem de $10 \%$.

Os resultados no ciclo WLTC foram um pouco melhores que os resultados do NEDC, mas ainda com THC e NMHC excedendo os limites. Aplicando o desconto de álcool não queimado, o NMHC-ETOH ficou com uma margem razoável abaixo do limite de NMCH. 
Estes dados estão ilustrados na Figura 7, onde é possível identificar os pontos críticos com mais facilidade. Estes pontos foram as emissões de THC no NEDC e WLTC, e de NMHC nos três ciclos. Sem descontar o álcool não queimado o NMHC não respeitaria os limites em nenhum dos ciclos.

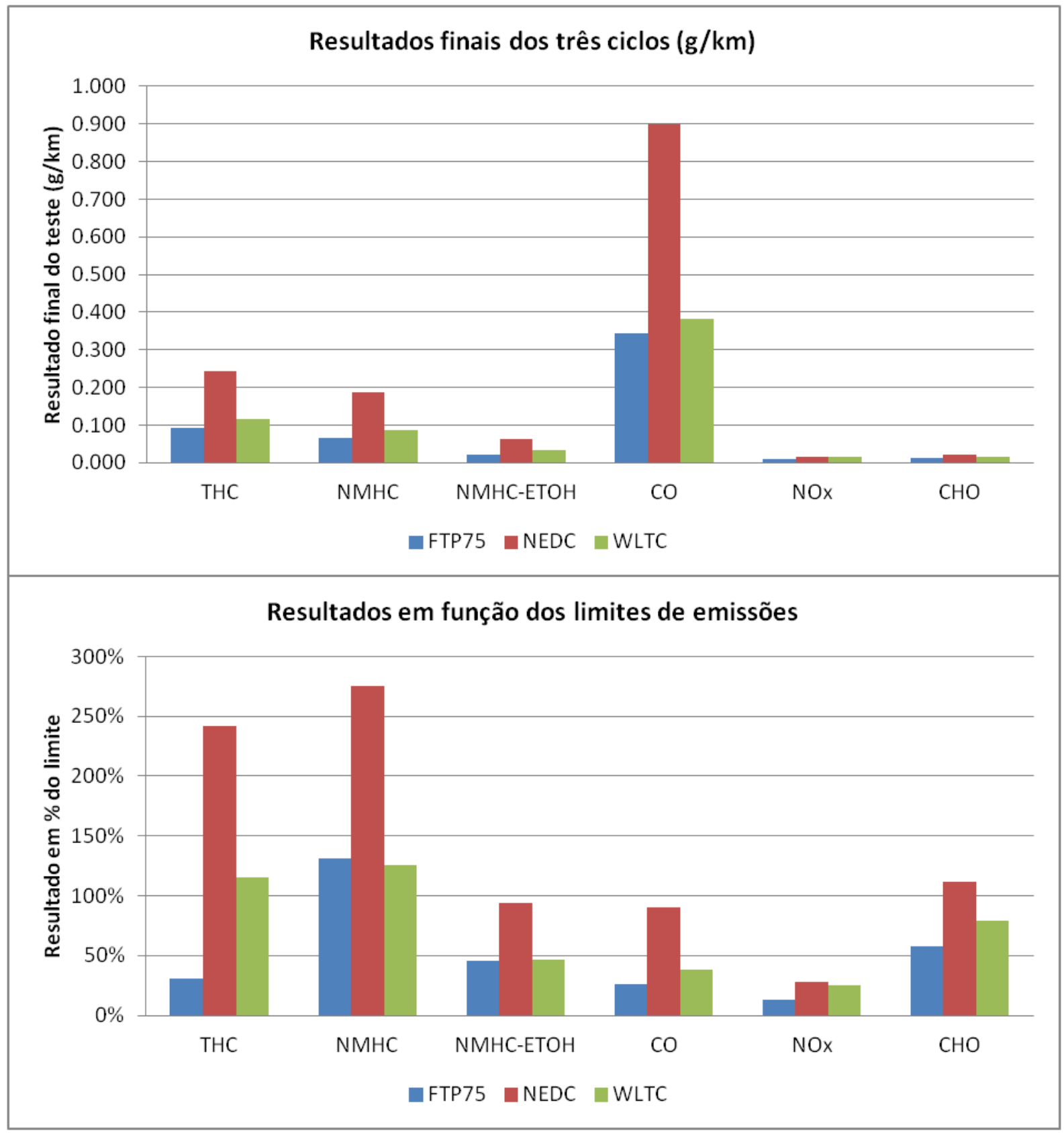

Figura 7: Representação dos resultados de emissões nos três ciclos

O efeito da ponderação reduzida na primeira fase do FTP75 pode ser observado na Figura 8. Sem a ponderação, o valor de NMHC ultrapassou $200 \%$ do limite legal, porém, com o desconto de álcool não queimado, o NMHC-ETOH ficou pouco abaixo dos $70 \%$. O poluente 
mais perto do limite legal foi o $\mathrm{CHO}$, com $88.5 \%$ do limite legal. Apesar do aumento dos resultados, os mesmos ainda seriam suficientes para respeitar a PL6.

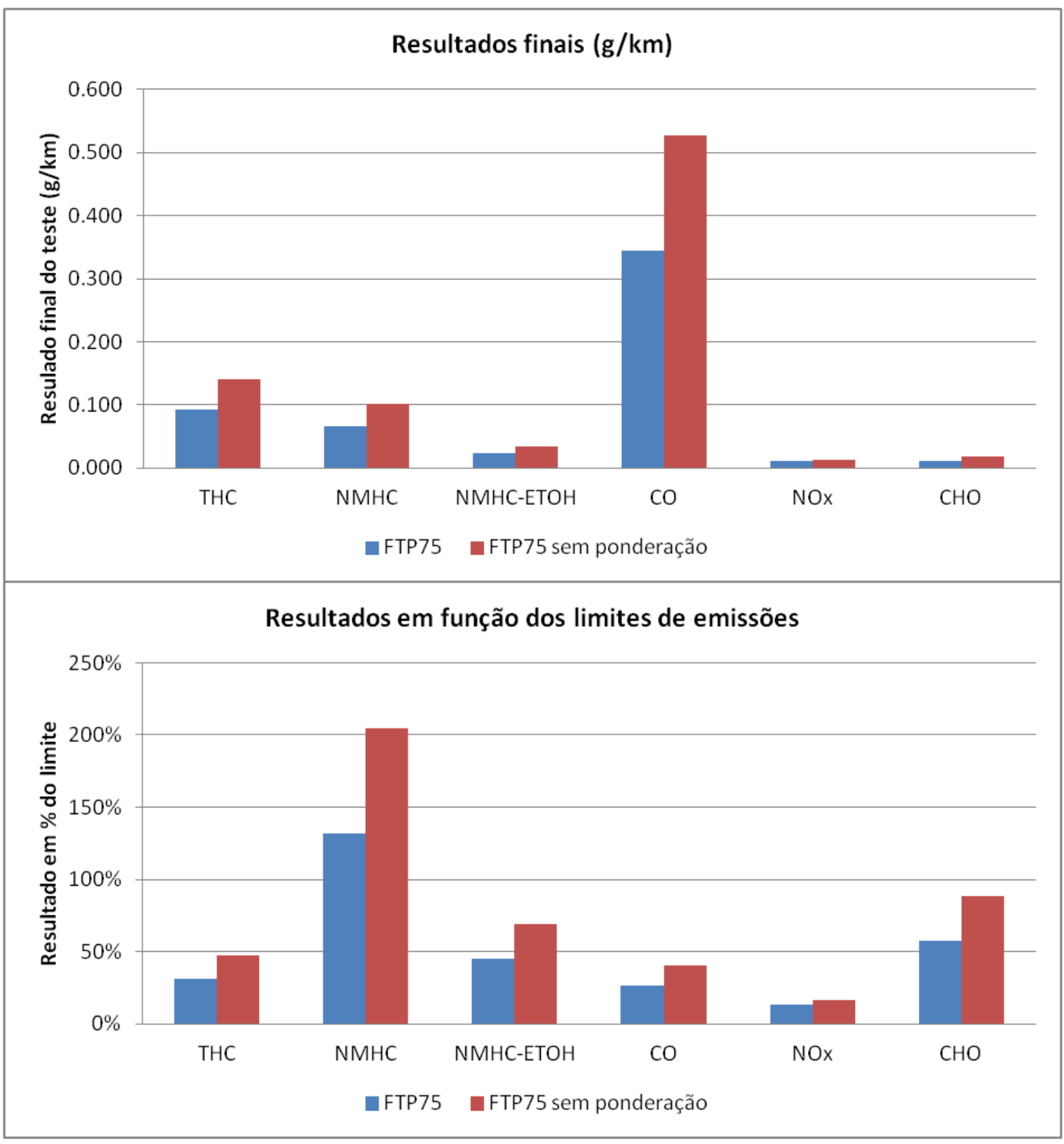

Figura 8: Comparação de resultados do FTP75 com e sem fatores de ponderação por fase

Assim como nos testes com E22, a maior parte das emissões ocorre nos primeiros segundos dos ciclos. A fase inicial dos ciclos pode ser observada na Figura 9. Nos três testes, o analisador de THC atingiu o fundo de escala de 10000 ppm. No NEDC o catalisador demora mais tempo para aquecer e, portanto demora mais tempo para atingir sua eficiência máxima. 


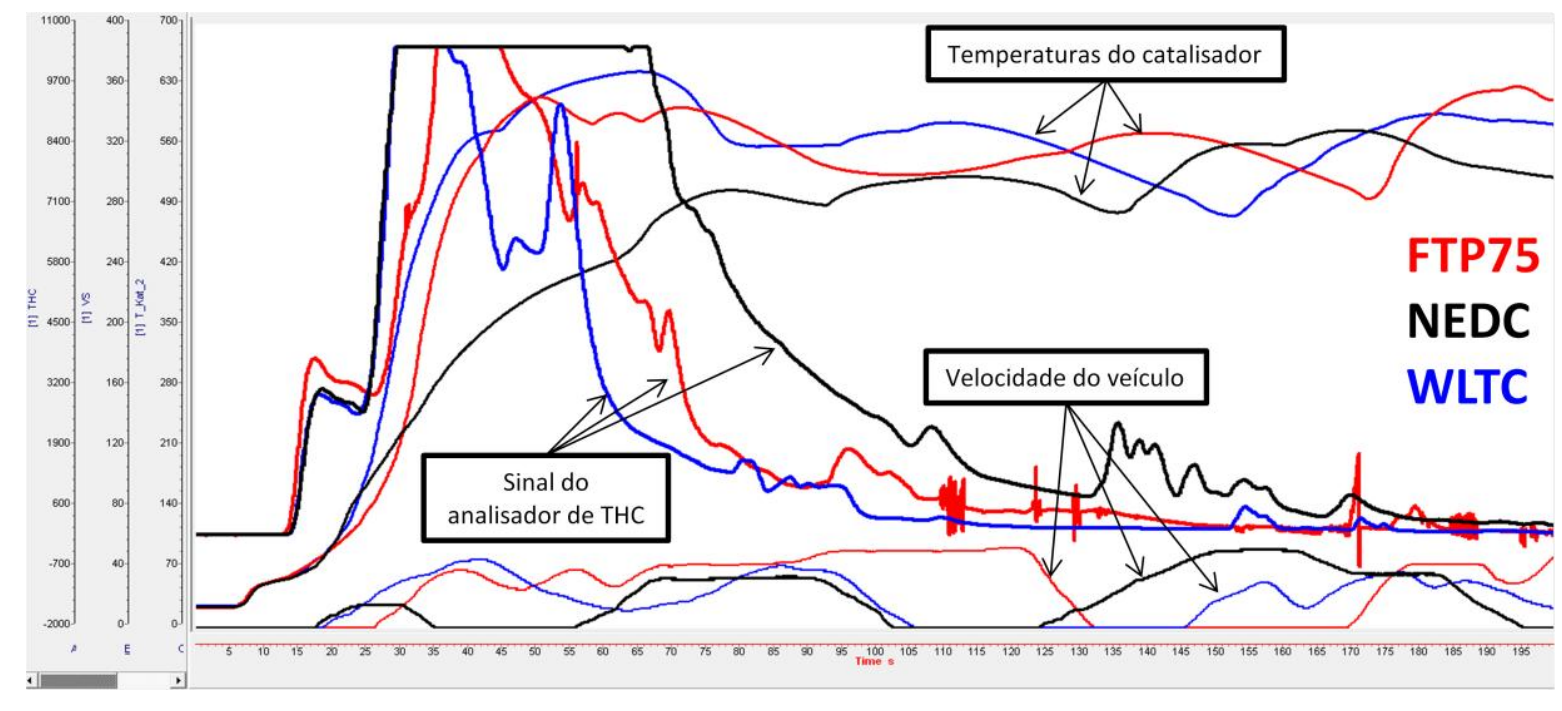

Figura 9: Comparação do modal entre os três ciclos nos primeiros 200 segundos de teste

Destacando as fases frias de cada ciclo, temos os resultados expressos em massa na Tabela 9. Como esperado, a maior parte das emissões acontece na fase fria. O NEDC foi o ciclo com maiores emissões de THC, NMHC e CO na fase fria. Entretanto, os desvios não são muito expressivos. Nota-se também que o CHO emitido durante o NEDC, apesar de ter o pior resultado final $(\mathrm{g} / \mathrm{km})$ e o pior resultado em função do limite fictício admitido, foi o poluente que teve a menor emissão em massa tanto na fase fria, quanto nas fases quentes. Isto evidencia, mais uma vez, a influência dos fatores de ponderação por fase e das distâncias dos ciclos. O FTP75 é favorecido em relação ao NEDC por ter fator de ponderação reduzido na primeira fase, e por ter uma distância maior. O WLTC também se favorece por ter a maior distância total dos três ciclos.

Tabela 9: Comparação de emissões em massa nas fases frias e quentes

\begin{tabular}{|l|c|c|c|c|c|c|}
\cline { 2 - 7 } \multicolumn{1}{c|}{} & \multicolumn{3}{c|}{ Fase Fria (I) } & \multicolumn{3}{c|}{ Fase Quente (II, III, IV) } \\
\cline { 2 - 7 } \multicolumn{1}{c|}{} & FTP75 & NEDC & WLTC & FTP75 & NEDC & WLTC \\
\hline Distância $(\mathrm{km})$ & 5.779 & 3.960 & 3.087 & 11.943 & 6.886 & 20.107 \\
\hline THC $(\mathrm{g})$ & 2.395 & 2.584 & 2.507 & 0.108 & 0.043 & 0.171 \\
\hline NMHC (g) & 1.794 & 2.023 & 1.947 & 0.018 & 0.010 & 0.036 \\
\hline NMHC- ETOH $(\mathrm{g})$ & 0.599 & 0.684 & 0.694 & 0.014 & 0.009 & 0.032 \\
\hline CO $(\mathrm{g})$ & 8.825 & 9.631 & 7.482 & 0.527 & 0.127 & 1.384 \\
\hline NOx $(\mathrm{g})$ & 0.165 & 0.160 & 0.203 & 0.066 & 0.019 & 0.145 \\
\hline CHO (g) & 0.306 & 0.238 & 0.268 & 0.008 & 0.003 & 0.019 \\
\hline
\end{tabular}


Nos casos do HC e do NMHC, que foram os gases com piores resultados no NEDC, observase que as emissões em massa na primeira fase não foram tão diferentes quanto os resultados finais. A Figura 10 mostra esta comparação. Os valores de THC e NMHC emitidos no NEDC foram cerca de 10\% superiores em relação ao FTP75 e 3.5\% superiores em relação ao WLTC. Descontando o álcool não queimado, as emissões de NMHC-ETOH nos três ciclos ficam muito semelhantes.

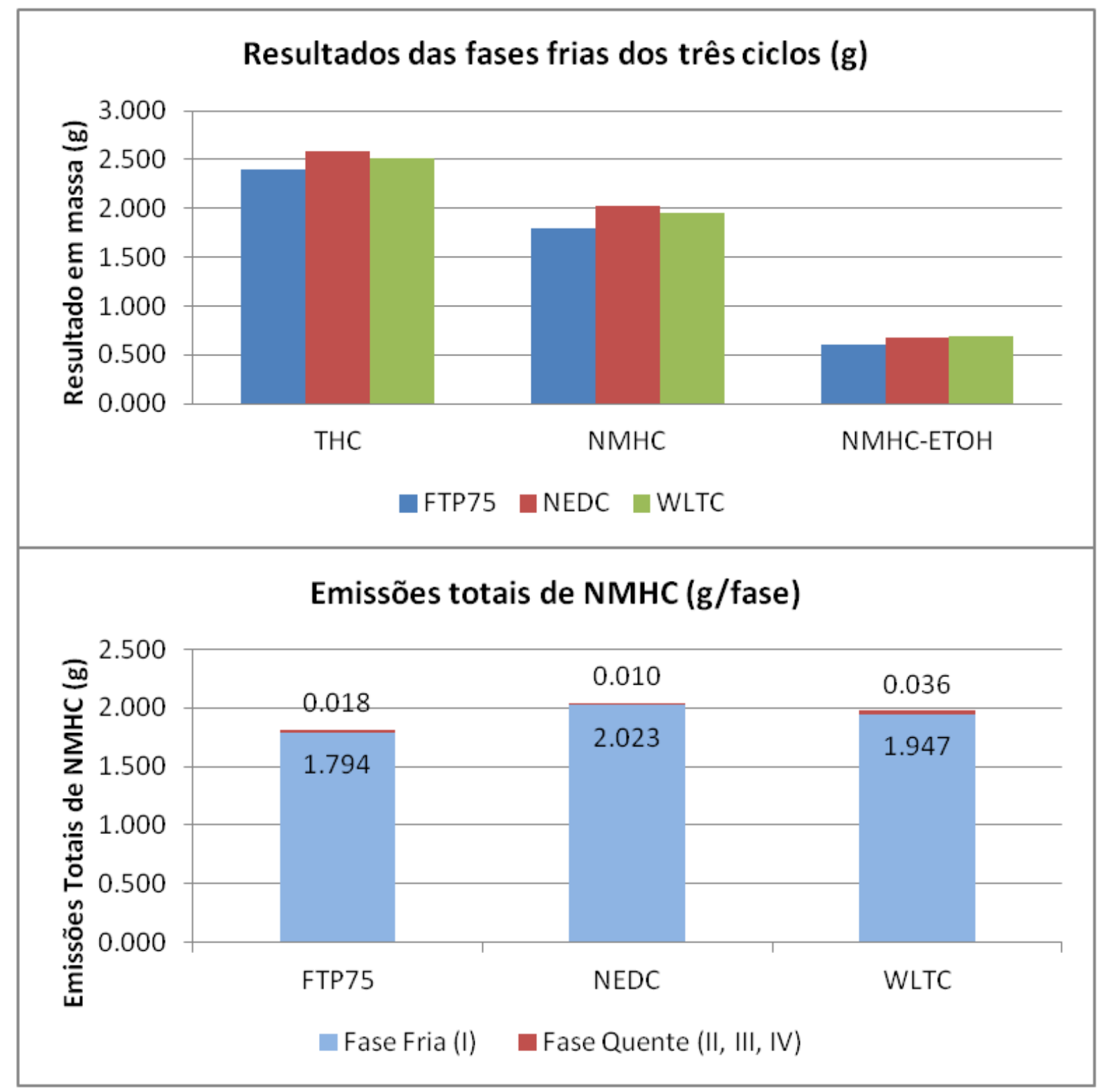

Figura 10: Emissões de THC, NMHC e NMHC-ETOH nas fases frias em massa

A proporção dos gases emitidos nas fases frias dos testes com E100 são ainda maiores que as proporções dos testes com E22. Estes dados estão descritos na Tabela 10. As emissões de NMCH na fase fria do FTP75 representam 99\% das emissões totais deste gás durante o teste 
inteiro. Mesmo assim, é permitido descontar o valor de álcool não queimado e ponderar a primeira fase do ciclo com um fator menor que nas outras duas fases.

Tabela 10: Proporção de massa emitida durantes fases frias

\begin{tabular}{|l|c|c|c|}
\cline { 2 - 4 } \multicolumn{1}{c|}{} & \multicolumn{3}{c|}{ Proporção da fase fria sobre os ciclos inteiros } \\
\cline { 2 - 4 } \multicolumn{1}{c|}{} & FTP75 & NEDC & WLTC \\
\hline Distância $(\mathrm{km})$ & $32.6 \%$ & $36.5 \%$ & $13.3 \%$ \\
\hline THC $(\mathrm{g})$ & $95.7 \%$ & $98.4 \%$ & $93.6 \%$ \\
\hline NMHC $(\mathrm{g})$ & $99.0 \%$ & $99.5 \%$ & $98.2 \%$ \\
\hline NMHC- ETOH $(\mathrm{g})$ & $97.7 \%$ & $98.7 \%$ & $95.6 \%$ \\
\hline CO $(\mathrm{g})$ & $94.4 \%$ & $98.7 \%$ & $84.4 \%$ \\
\hline NOx $(\mathrm{g})$ & $71.3 \%$ & $89.2 \%$ & $58.3 \%$ \\
\hline CHO $(\mathrm{g})$ & $97.6 \%$ & $98.7 \%$ & $93.3 \%$ \\
\hline
\end{tabular}

Nas fases quentes, as emissões em g/km são muito semelhantes para o NMHC e NMHCETOH. O NEDC leva uma pequena vantagem nas emissões de THC. Assim como nos testes com E22, o WLTC é o ciclo com mais emissões por quilômetro de CO.

Tabela 11: Resultados em massa e em g/km das fases quentes

\begin{tabular}{|l|c|c|c|c|c|c|}
\cline { 2 - 7 } \multicolumn{1}{c|}{} & \multicolumn{3}{c|}{ Fase Quente (II, III, IV) - (g) } & \multicolumn{3}{c|}{ Fase Quente (II, III, IV) - (g/km) } \\
\cline { 2 - 7 } \multicolumn{1}{c|}{} & FTP75 & NEDC & WLTC & FTP75 & NEDC & WLTC \\
\hline THC & 0.108 & 0.043 & 0.171 & 0.009 & 0.006 & 0.009 \\
\hline NMHC & 0.018 & 0.010 & 0.036 & 0.002 & 0.001 & 0.002 \\
\hline NMHC- ETOH & 0.014 & 0.009 & 0.032 & 0.001 & 0.001 & 0.002 \\
\hline CO & 0.527 & 0.127 & 1.384 & 0.044 & 0.018 & 0.069 \\
\hline NOx & 0.066 & 0.019 & 0.145 & 0.006 & 0.003 & 0.007 \\
\hline CHO & 0.008 & 0.003 & 0.019 & 0.001 & 0.000 & 0.001 \\
\hline
\end{tabular}




\section{CONCLUSÃO}

Na comparação inicial das características dos ciclos, é evidente que o WLTC tende a ser o ciclo mais desafiador dentre os três. O NEDC, por ter perfis de velocidade constante e poucas condições dinâmicas, que não representam condições reais de condução, apresenta-se como um ciclo menos exigente.

Os resultados finais dos testes mostram que estas impressões iniciais estavam erradas. Como os resultados são dados em $\mathrm{g} / \mathrm{km}$, o NEDC, que é o ciclo com menor distância total, tem resultados finais maiores que o FTP75 e o WLTC. Assim, o NEDC acaba sendo o ciclo mais severo entre os três.

Na comparação de emissões em massa, nota-se que tanto com E22, quanto com E100, os valores em massa das poluentes mais críticos (THC e NMHC) foram semelhantes nos três ciclos. Sendo a fase fria responsável pela maior parte das emissões nos três ciclos, as distâncias de cada ciclo e os fatores de ponderação do FTP75 distorcem os resultados finais. Já as emissões das fases quentes, após o catalisador atingir sua máxima eficiência, são praticamente proporcionais às distâncias percorridas, e o resultado em $\mathrm{g} / \mathrm{km}$ fica mais próximo entre os três ciclos. Seguindo estas observações, faz mais sentido analisar as fases frias de cada ciclo expressas em massa (g), e analisar as fases quentes em massa por distância $(\mathrm{g} / \mathrm{km})$. Encarando os resultados dos três ciclos desta forma, não houve diferenças expressivas nos resultados e, portanto, a calibração feita para o FTP75 foi capaz de manter um nível de emissões semelhante nos três ciclos.

Apesar deste nível de emissões manter-se estável na comparação entre os ciclos, se este veículo brasileiro fosse submetido à legislação europeia, ele ficaria ligeiramente abaixo dos limites com E22. Entretanto, com E100 os níveis de emissões de THC e NMHC (sem o desconto de álcool não queimado) ficariam bem acima dos limites EURO6 (0.100 g/km de THC e $0.068 \mathrm{~g} / \mathrm{km}$ de NMHC).

As grandes diferenças nos resultados finais se devem às diferenças de distância entre os ciclos e aos métodos utilizados para cálculo destes resultados. O peso menor na primeira fase do FTP75 e o desconto de álcool não queimado disfarçam o desempenho dos veículos homologados no Brasil. Para adequar-se aos padrões internacionais seriam necessárias alterações na legislação que reduzam os limites de emissões de poluentes. 


\section{REFERÊNCIAS}

[1] KÜHLWEIN, Jörg; GERMAN, John; BANDIVADEKAR, Anup. Development Of Test Cycle Conversion Factors Among Worldwide Light-Duty Vehicle Co2 Emission

Standards. The International Council on Clean Transportation, September 2014.

[2] DAHAM, Basil. Drive Cycles. Disponível em:

http://www.daham.org/basil/leedswww/emissions/drivecycles.htm. Acesso em: 26/05/2016.

[3] Continental Automotive GmbH Powertrain Division. Worldwide Emission Standards and Related Regulations. Regensburg, March 2015.

[4] Associação Brasileira de Normas Técnicas. NBR 6601 Veículos rodoviários

automotores leves - Determinação de hidrocarbonetos, monóxido de carbono, óxidos de nitrogênio, dióxido de carbono e material particulado no gás do escapamento. Rio de Janeiro, 18/09/2012. 